\title{
Factors associated with inconsistency in self-reported mild traumatic brain injury over time among military personnel in Iraq
}

Nathaniel W. Nelson, Carolyn R. Anderson, Paul Thuras, Shannon M. Kehle-Forbes, Paul A. Arbisi, Christopher R. Erbes and Melissa A. Polusny

\section{Background}

Estimates of the prevalence of mild traumatic brain injury (mTBI) among military personnel and combat veterans rely almost exclusively on retrospective self-reports; however, reliability of these reports has received little attention.

\section{Aims \\ To examine the consistency of reporting of mTBI over time and identify factors associated with inconsistent reporting. \\ Method \\ A longitudinal cohort of 948 US National Guard Soldiers deployed to Iraq completed self-report questionnaire screening for mTBI and psychological symptoms while in-theatre 1 month before returning home (time $1, T_{1}$ ) and 1 year later (time $2, T_{2}$ ).}

\section{Results}

Most respondents $(n=811,85.5 \%)$ were consistent in their reporting of $\mathrm{mTBI}$ across time. Among those who were inconsistent in their reports $(n=137,14.5 \%)$, the majority denied $\mathrm{mTBI}$ at $T_{1}$ and affirmed $\mathrm{mTBI}$ at $T_{2}(n=123,89.8 \%)$. Respondents rarely endorsed $\mathrm{MTBI}$ in-theatre and later denied $\mathrm{mTBI}(n=14,10.2 \%$ of those with inconsistent reports). Post-deployment post-traumatic stress symptoms and non-specific physical complaints were significantly associated with inconsistent report of MTBI.

\section{Conclusions}

Military service members' self-reports of mTBI are generally consistent over time; however, inconsistency in retrospective self-reporting of MTBI status is associated with current posttraumatic stress symptoms and non-specific physical health complaints

\section{Declaration of interest}

C.R.A., P.T., S.M.K.-F., P.A.A, C.R.E. and M.A.P. are US government employees. The views expressed in this article are those of the authors and do not reflect the official policy or position of the US Department of Veterans Affairs, US Department of the Army or US Department of Defense.
Traumatic brain injury (TBI) is regarded as one of the most common combat-related injuries encountered by military personnel who have served in the conflicts in Iraq (Operation New Dawn, OND; previously Operation Iraqi Freedom, OIF) and Afghanistan (Operation Enduring Freedom, OEF). ${ }^{1-3}$ In fact, it is possible that TBI has transpired with greater regularity in OEF/OIF than in any previous war or conflict. Mild traumatic brain injury (mTBI or concussion) is commonly defined as a traumatically induced physiological disruption of brain functioning because of an external force that is accompanied by at least one of the following symptoms/signs: loss of consciousness that persists for no longer than $30 \mathrm{~min}$, no more than $24 \mathrm{~h}$ period of post-traumatic amnesia, altered mental status (dizziness, confusion, disorientation), and/or focal neurologic deficits. ${ }^{4}$ This injury is thought to be especially common in OEF/OIF samples related to frequent reports of blast exposure associated with improvised explosive devices (IEDs) and other novel explosive mechanisms. ${ }^{5}$ As common as mTBI is believed to be, precise prevalence in OEF/OIF is not clear. Rates of MTBI reported in past surveys have varied from as low as 9 to $12 \%{ }^{1-3}$ to as high as 19 to $23 \%{ }^{3,6,7}$ Several factors may underlie these varying rates of self-reported mTBI in OEF/OIF personnel, such as: the extent of combat participation by cohort; level of scrutiny employed in defining $\mathrm{mTBI}^{8}$ the specific mTBI screening or assessment tool that is implemented; ${ }^{9-12}$ measurement error and equivocal psychometric properties of contemporary mTBI screening tools; ${ }^{13-15}$ and timing of mTBI screening assessments.

Psychological distress may also influence self-report of mTBI among OEF/OIF combat veterans. Unlike civilian mTBI samples, whose injuries do not typically result in extended emotional difficulties, such as depression or anxiety, ${ }^{16}$ OEF/OIF personnel who sustain $\mathrm{mTBI}$ as a result of combat are often at increased risk of developing psychiatric difficulties, such as post-traumatic stress symptoms (PTSS). ${ }^{1}$ Recollection and reports of previous trauma events are not static and often vary with current psychological state and health perceptions. ${ }^{17}$ Studies have shown that retrospective report of perceived threats following exposure to natural disaster ${ }^{18}$ and deployment-related stressors ${ }^{17,19}$ may become amplified with chronic PTSS over time. For example, one study found that stability of self-reports of peritraumatic responses were poor among individuals whose PTSS did not remit within the first year of trauma exposure relative to those without PTSS or whose symptoms did remit over time. ${ }^{20}$ Although we are not aware of any study that has explicitly examined whether PTSS or formally diagnosed post-traumatic stress disorder (PTSD) predicts consistency in self-reported mTBI over time, it is conceivable that chronic PTSS, depression and other forms of emotional distress that persist or develop during the post-deployment phase contribute to attribution errors or biases. ${ }^{11}$ Such attribution errors may result in retrospective endorsement of mTBI on post-deployment screening. For instance, subjective cognitive limitations and other post-deployment 'post-concussive' symptoms might be retrospectively attributed to combat-related mTBI, even if symptoms represent PTSS $^{3}$ or symptoms of depression. ${ }^{1}$ Non-specific and medically unexplained physical symptoms (such as fatigue, headache, tinnitus) are also very common following war and terroristic events. ${ }^{21}$ Confronted with the ambiguous nature of these physical symptoms, returning service members and veterans may be drawn to discrete, identifiable explanations or causes, such as mTBI, even if 
psychological mechanisms play an important role in their onset, severity and persistence. Secondary gain issues may also affect self-report of mTBI during the post-deployment transition phase. ${ }^{11,22-24}$ Disability income related to deployment-related injuries, including mTBI, is substantial, ${ }^{25}$ and recent studies have suggested that symptom exaggeration and/or cognitive feigning transpires at concerning rates in OEF/OIF mTBI samples, especially among those evaluated in the compensation and pension disability context. ${ }^{23,24}$ Post-deployment disability claims initiated in relation to combat-related mTBI or other physical injury may modify self-perceptions of combat events (such as blast exposures) that were not previously regarded as significant. Secondary gain issues may be particularly relevant if an individual experiences significant income distress during post-deployment, whereby disability income is identified as necessary to meet basic survival needs.

In a previous longitudinal study of self-reported mTBI in OIF military personnel, ${ }^{3}$ we surveyed a cohort of National Guard Soldiers 1 month before their return from Iraq (time $1, T_{1}$ ) and 1 year after return from deployment (time $2, T_{2}$ ). At $T_{1}, 9 \%$ of respondents endorsed a history of $\mathrm{mTBI}$ as evidenced by an injury that reportedly resulted in loss of consciousness or altered mental status, a finding that was relatively comparable with some survey reports. ${ }^{1,2}$ By contrast, rates of self-reported mTBI at $T_{2}$ increased to $22 \%$, a rate that is similar to those of other survey reports. ${ }^{6,7}$ This greater than twofold increase in self-reported mTBI supported the notion that timing of mTBI screening has a significant effect on rates of self-reported injury. However, psychological and health outcomes in our initial study were examined as a function of mTBI status at $T_{1}$ only (i.e. 1 month before returning from Iraq); factors associated with inconsistency in self-reported mTBI at $T_{2}$ were not investigated. The aims of the current study were to examine consistency of reporting of mTBI over time (whether respondents consistently endorsed mTBI or did not endorse mTBI at both $T_{1}$ and $T_{2}$ ) and investigate factors related to inconsistency of self-reported mTBI over time (mTBI not endorsed at $T_{1}$ but endorsed at $T_{2}$ ). We hypothesised that $T_{2}$ PTSS and depression symptoms, $T_{2}$ non-specific physical complaints (such as fatigue, headache, tinnitus), active disability claim and current income distress would emerge as significantly associated with inconsistent reports of mTBI between $T_{1}$ and $T_{2}$ (i.e. not endorsing mTBI in-theatre and endorsing mTBI 1 year post-deployment).

\section{Method}

\section{Procedures and participants}

A full description of the study methods has been published elsewhere ${ }^{3}$, and is briefly summarised here. Data were examined from two waves of a larger, prospective, longitudinal investigation of post-deployment mental health in a sample of US Army National Guard Soldiers deployed to Iraq from March 2006 to July 2007. $T_{1}$ data were collected approximately 1 month prior to soldiers' return from a 16-month combat deployment. While attending a redeployment transition briefing, all soldiers from a brigade combat team were invited to participate in the study and given a packet containing an informed consent document, brief paper-and-pencil survey that screened for blast exposure, mTBI and psychiatric symptoms (PTSS and depression), and a return envelope. Soldiers were asked to seal their completed or uncompleted surveys in envelopes, which were collected by the onsite military collaborator and delivered to the research team.
$T_{2}$ data were collected 1 year following soldier return from deployment using standard mailed survey procedures. Soldiers were sent a follow-up packet containing an informed consent document (cover letter), paper-and-pencil survey that included questions about deployment-related experiences, including exposure to explosive blasts and injuries sustained during deployment, history of mTBI during deployment and current psychiatric symptoms, postage paid return envelope and $\$ 20$ incentive. Non-responders were sent reminders to encourage participation.

As shown in Fig. 1, a total of 2677 soldiers completed $T_{1}$ surveys, of which 1935 participants provided contact information and agreed to be followed for participation in future studies (43 participants were lost to follow-up including 2 deceased; 4 temporarily away or incarcerated; 20 were redeployed; 26 had untrackable addresses, or some combination of these). Of the 1892 participants surveyed, a total of 953 (50.4\%) participants completed the $T_{2}$ survey. The final analysis included 948 soldiers who completed questionnaires at both $T_{1}$ and $T_{2}$ (1 participant was removed from analysis because of reporting loss of consciousness $>20$ min and may have sustained traumatic brain injuries that were greater than mild in severity; 4 participants were missing $T_{1}$ screening data). Participation rates were nearly identical between those who endorsed mTBI $(34.8 \%)$ and those who did not endorse mTBI $(35.9 \%)$ at $T_{1}, \chi^{2}(1)=0.12, P=0.73$.

Compared with those who completed the $T_{2}$ follow-up questionnaire, $T_{2}$ survey non-responders were younger (mean age 29.06, s.d. $=7.70 \quad$ v. mean age 31.47 , s.d. $=8.31$ ), $t(2541)=-7.36, \quad P<0.001)$, more likely to be unmarried (62.3\% v. $51.2 \%, P<0.001)$, more likely to be of enlisted rank $(91.6 \%$ v. $86.6 \%, P<0.001)$ and had lower levels of in-theatre depression (mean 8.53 , s.d. $=7.66$ v. mean 9.28, s.d. $=8.43$ ), $t(2615)=2.25, P=0.025$.

\section{Measures}

\section{mTBI screening}

At $T_{1}$, blast exposure and mTBI history were assessed with an adaptation of the Defense and Veterans Brain Injury Center (DVBIC) screening tool. ${ }^{12}$ Accordingly, participants were determined to have a positive mTBI history at $T_{1}$ if they reported sustaining any injury during deployment and endorsed the following item assessing the presence of altered mental status or loss of consciousness: 'Did any injury cause you to be dazed/ confused, "see stars", get knocked out, or lose consciousness?.

At $T_{2}$, criteria for mTBI status included the same screening information as was obtained at $T_{1}$ (i.e. dazed/confused, 'see stars', knocked out or loss of consciousness). However, further acutestage injury information was also obtained to further inform the likelihood that participants sustained mTBI and to ensure that these events were no greater than mild in severity. Specifically, at $T_{2}$, participants were assigned a positive mTBI history if they endorsed at least one of the items from the following relevant to altered mental status or loss of consciousness: 'Did any injury received while you were deployed result in any of the following: (1) being dazed, confused, or "seeing stars"; (2) not remembering the injury; (3) losing consciousness (knocked out) for less than a minute; or (4) losing consciousness for 1 to 20 minutes'.

\section{Assessment of independent variables}

We assessed PTSS at $T_{1}$ and $T_{2}$ using the PTSD Checklist-Military (PCL-M). ${ }^{26}$ Depressive symptoms were assessed at $T_{1}$ and $T_{2}$ using the Beck Depression Inventory (BDI-II). ${ }^{27}$ Generalised somatic symptoms were assessed at $T_{2}$ using the 15-item somatic 


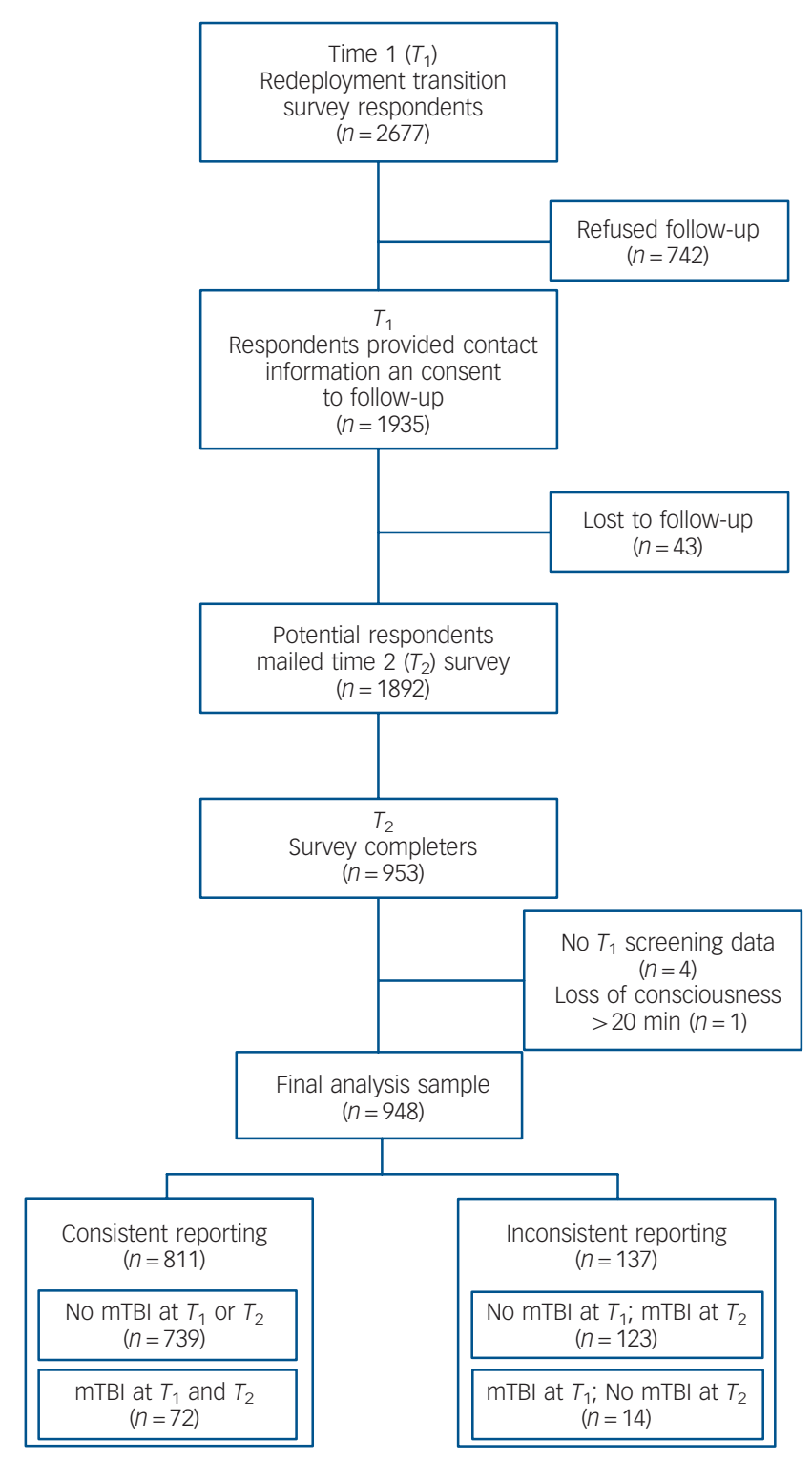

Fig. 1 Flow of participations.

mTBI, mild traumatic brain injury. and analysis of variance were used to examine differences between the groups on continuous symptom measures at $T_{1}$ and $T_{2}$. Logistic regression analyses with forced entry was used to examine the role of various independent variables in determining the likelihood of being in the No TBI-TBI reporting group, with the No TBI-No TBI as the referent group. The use of forced entry (as opposed to stepwise entry) regression allowed for the simultaneous evaluation of the independent contribution of all independent variables to the prediction. All continuous measures were converted to standard scores prior to entry in the regression model. All analyses were conducted using SPSS Version 19 for Windows.

\section{Results}

\section{Sample characteristics}

Demographic characteristics of the sample are reported in Table 1. A comparison of the No TBI-No TBI and No TBI-TBI groups found no significant differences on age, marital status, ethnicity, education, income distress or prior deployment. The No TBI-TBI group had a higher proportion of men $(97.5 \%$ v. 91.0\%; $\left.\chi^{2}(1)=5.77, \quad P=0.016\right)$, enlisted soldiers $(93.4 \%$ v. $85.0 \%$; $\left.\chi^{2}(1)=7.49, P=0.006\right)$, and reports of disability claims $(56.7 \% v$. $\left.38.4 \% ; \chi^{2}(1)=13.78, P<0.001\right)$ than did the No TBI-No TBI group.

\section{Consistency of $\mathrm{mTBI}$ reporting over time}

Among those who were inconsistent in their reports of mTBI over time $(n=137,14.5 \%)$, the vast majority $(n=123,89.8 \%)$ did not endorse mTBI at $T_{1}$ and endorsed mTBI at $T_{2}$. Only 14 respondents (10.2\% of those with inconsistent reports) endorsed mTBI at $T_{1}$ and did not endorse mTBI at $T_{2}$. Given the small size of this latter group, these participants were excluded from further analyses. Demographic characteristics of consistent (No TBI-No TBI) and inconsistent (No TBI-TBI) respondents are presented in Table 2. Compared with the consistent (No TBI-No TBI) group, soldiers with inconsistent reports (No TBI-TBI) endorsed significantly greater depression $\left(T_{1}\right.$ and $\left.T_{2}\right)$, PTSS $\left(T_{1}\right.$ and $\left.T_{2}\right)$, and somatic complaints $\left(T_{2}\right)$. The No TBI-TBI group was also more likely to report an active disability claim than the No TBI-No TBI group, although income distress was not significantly different between groups.

\section{Factors associated with inconsistency in $\mathrm{MTBI}$ endorsement}

Next, we examined factors associated with change in mTBI status from $T_{1}$ (No TBI) to $T_{2}$ (TBI). We used logistic regression to examine the significance of independent variables of being in the No TBI-TBI group with the No TBI-No TBI serving as the reference group. Variables were selected for the regression based on significant differences between the groups in univariate comparisons. Standard scores for BDI-II and PCL-M scores at both $T_{1}$ and $T_{2}$, PHQ-15 scores at $T_{2}$, and indicators of Veterans Affairs disability claim and income distress were entered into the regression. Results are presented in Table 3. $T_{2}$ PCL-M and PHQ-15 scores were associated with membership in the No TBI-TBI group compared with the reference group. $T_{2}$ PCL-M was significantly associated with inconsistent reports of mTBI, demonstrating that the No TBI-TBI group endorsed significantly greater PTSD symptoms over time relative to the reference group. Disability claim status and income distress were not associated with change in self-reported mTBI status. 


\begin{tabular}{|llc|}
\hline $\begin{array}{lll}\text { Table } 1 \text { Demographic characteristics } \\
\text { Characteristics }\end{array}$ & $n$ \\
\hline Age in years, mean (s.d.) & $31.6(8.3)$ & 948 \\
\hline Male gender, $n$ (\%) & $870(92.8)$ & 938 \\
\hline Married status, $n$ (\%) & $536(56.8)$ & 943 \\
\hline White, $n$ (\%) & $820(88.1)$ & 931 \\
\hline High school education or less, $n(\%)$ & $199(21.1)$ & 944 \\
\hline Enlisted, $n$ (\%) & $810(86.4)$ & 937 \\
\hline Previous deployment, $n$ (\%) & $113(12.0)$ & 945 \\
\hline
\end{tabular}

\section{Interim severity of psychiatric symptoms across mTBI groups}

Figures 2 and 3 depict mean PTSS and depression symptom severity scores across the No TBI-TBI and reference groups. The two groups differed significantly on mean levels of PTSS and depression at both $T_{1}$ and $T_{2}$ (Table 2), with the No TBI-TBI group endorsing significantly higher symptoms on both measures at both $T_{1}$ and $T_{2}$ than the reference group. In addition, the increase in both PTSS $\left(F(1,848)=34.5, \quad P<0.001, \eta^{2}\right.$ and depression symptoms $\left(F(1,836)=11.5, P=0.001, \eta^{2}=0.014\right)$ from

\begin{tabular}{|c|c|c|c|c|c|c|}
\hline & \multicolumn{2}{|c|}{ mTBI self-report category } & \multicolumn{4}{|c|}{ Test statistics } \\
\hline & No TBI-No TBI $(n=739)$ & No TBI-TBI $(n=123)$ & F & $\chi^{2}$ & $P$ & $\eta^{2}$ \\
\hline \multicolumn{7}{|l|}{$T_{1}$} \\
\hline Post Traumatic Checklist-Military Version, mean (s.d.) & $29.5(10.8)$ & $34.8(13.6)$ & 22.7 & & $<0.001$ & 0.03 \\
\hline Beck Depression Inventory-II, mean (s.d.) & $7.9(7.3)$ & $10.9(8.3)$ & 16.6 & & $<0.001$ & 0.02 \\
\hline \multicolumn{7}{|l|}{$T_{2}$} \\
\hline Post Traumatic Checklist-Military Version, mean (s.d.) & $31.5(13.1)$ & 44.1 (15.4) & 95.0 & & $<0.001$ & 0.10 \\
\hline Beck Depression Inventory-II, mean (s.d.) & $9.9(8.8)$ & $16.1(9.0)$ & 51.2 & & $<0.001$ & 0.06 \\
\hline Patient Health Questionnaire-15, mean (s.d.) & $9.9(6.0)$ & $15.1(6.0)$ & 77.6 & & $<0.001$ & 0.08 \\
\hline Income distress, ${ }^{\mathrm{b}} \mathrm{n}(\%)$ & $61 / 730(8.4)$ & 15/119 (12.6) & & 2.2 & 0.14 & 0.05 \\
\hline Disability claim, $n(\%)$ & 279/727 (38.4) & $68 / 120(56.7)$ & & 13.8 & $<0.001$ & 0.13 \\
\hline
\end{tabular}

a. No TBI-No TBI includes respondents who denied mTBI history 1 month before return from deployment at time $1\left(T_{1}\right)$ and 1 year post-deployment (time 2, $T_{2}$ ). No TBI-TBI includes respondents who denied history of mild traumatic brain injury at $T_{1}$ and affirmed $\mathrm{mTBI}$ at $T_{2}$.

b. Income distress: responded 'Not at all' to question on the World Health Organization Quality of Life (WHO-BREF QOL), 'Do you have enough money to meet your needs?'.

\begin{tabular}{|c|c|c|c|}
\hline & $\beta$ (s.e.) & Wald & OR $(95 \% \mathrm{Cl})$ \\
\hline \multicolumn{4}{|l|}{$T_{1}$} \\
\hline Post Traumatic Checklist-Military Version & $-0.11(-0.17)$ & 0.41 & $0.90(0.64-1.25)$ \\
\hline Beck Depression Inventory-II & $0.12(0.17)$ & 0.49 & $1.13(0.81-1.57)$ \\
\hline \multicolumn{4}{|l|}{$T_{2}$} \\
\hline Post Traumatic Checklist-Military Version & $0.59(0.19)$ & $9.40 *$ & $1.81(1.24-2.64)$ \\
\hline Beck Depression Inventory-II & $-0.17(0.19)$ & 0.79 & $0.84(0.58-1.23)$ \\
\hline Patient Health Questionnaire-15 & $0.49(0.16)$ & $9.51^{*}$ & $1.63(1.20-2.22)$ \\
\hline Disability claim & $-0.20(0.22)$ & 0.79 & $0.82(0.53-1.27)$ \\
\hline Income distress ${ }^{b}$ & $0.51(0.37)$ & 1.86 & $1.67(0.80-3.50)$ \\
\hline Constant & $-2.40(0.39)$ & 37.15 & 0.09 \\
\hline
\end{tabular}

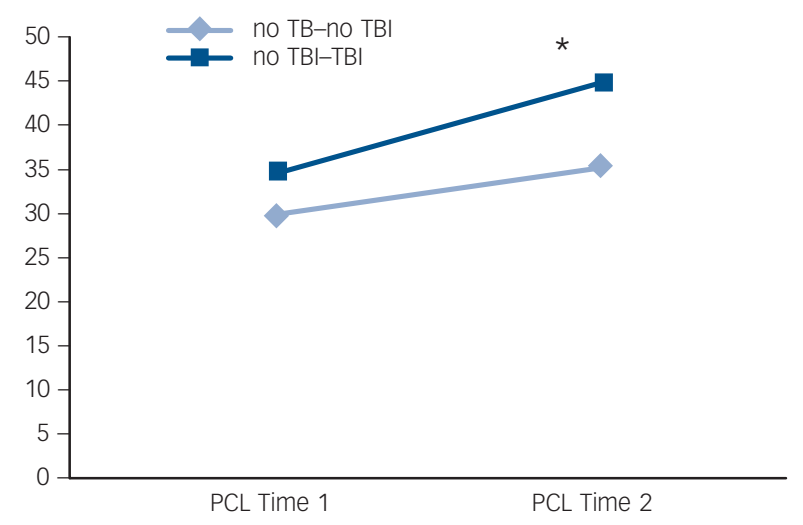

Fig. 2 Mean symptoms of post-traumatic stress symptoms (PTSS) endorsed in-theatre (Time 1, $T_{1}$ ) and 1 year post-deployment (Time $2, T_{2}$ ) across mild traumatic brain injury (mTBI) groups.

'No TBI-No TBI' ( $n=739$ ) includes respondents who denied mTBI history 1 month before return from deployment $\left(T_{1}\right)$ and 1 year post-deployment $\left(T_{2}\right)$. 'No TBI-TBI' $(n=123)$ includes respondents who denied history of mTBI at $T_{1}$ and affirmed mTBI at $T_{2}$. PCL-M, Post Traumatic Checklist - Military Version. ${ }^{*} P<0.001$.

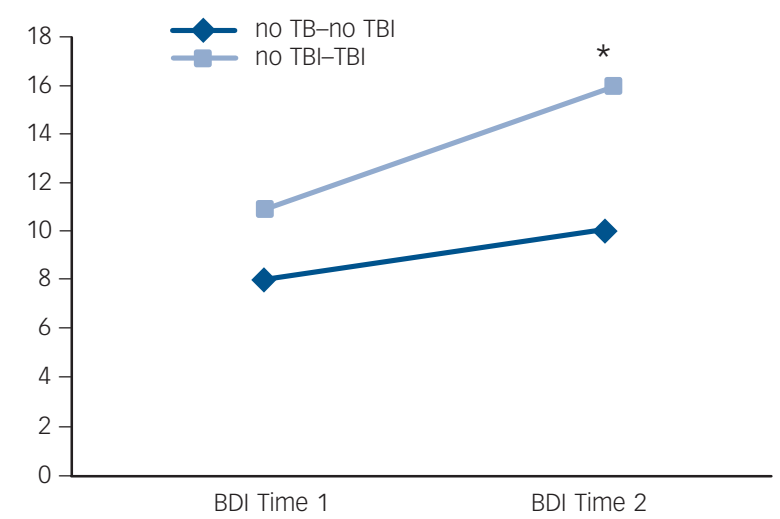

Fig. 3 Mean depressive symptoms endorsed in-theatre (Time 1, $T_{1}$ ) and 1 year post-deployment (Time 2, $T_{2}$ ) across mild traumatic brain injury (mTBI) groups.

'No TBI-No TBI' ( $n=739$ ) includes respondents who denied mTBI history 1 month before return from deployment $\left(T_{1}\right)$ and 1 year post-deployment $\left(T_{2}\right)$. 'No TBI-TBI' $(n=123)$ includes respondents who denied history of mTBI at $T_{1}$ and affirmed mTBI at $T_{2}$. BDI, Beck Depression Inventory-II. * $P<0.001$. 
$T_{1}$ to $T_{2}$ was significantly greater for the No TBI-TBI group relative to the No TBI-No TBI group.

\section{Discussion}

Although there is little question that TBI is a major health concern among OEF/OIF soldiers, the assertion that TBI represents a 'signature' injury of the wars in Iraq and Afghanistan is warranted only to the extent that occurrence is reliably known. To our knowledge, the current study is the first to examine factors that are significantly associated with consistency and inconsistency in self-reported combat-related mTBI over time. Consistent with retrospective reports of trauma exposure, the majority of respondents $(85.5 \%)$ demonstrated stable endorsements of combatrelated mTBI over time. A meaningful minority of respondents demonstrated inconsistent self-reports of mTBI (14.5\%), with most inconsistency involving not reporting mTBI while in-theatre and later affirming a history of mTBI post-deployment. We hypothesised that inconsistency in reporting of mTBI history (not endorsing mTBI in-theatre and endorsing mTBI 1 year post-deployment) would be associated with severity of postdeployment PTSS, depression, non-specific physical complaints, as well as secondary gain issues (disability claim status, income distress). In partial support of this hypothesis, results showed that change in self-report of mTBI history over time was associated with higher post-deployment PTSS and non-specific physical complaints, after controlling for in-theatre levels of trauma and depression symptoms. Inconsistent reporting of mTBI was also associated, at least on a univariate level, with having submitted a disability claim, which raises the possibility that the endorsement of mTBI at $T_{2}$ was, at least for some respondents, associated with secondary gain issues as has been reported in previous studies. ${ }^{23,24}$ However, disability claim status was not significantly associated with inconsistent reports of mTBI when PTSS, depression and physical complaints were accounted for.

Stability of self-reported trauma exposure tends to vary with psychological state and perception of health ${ }^{17}$ and retrospective report of perceived threats may become amplified with chronic PTSS over time. ${ }^{17-20}$ In this context, current findings offer preliminary support to the notion that a similar process is taking place with reports of mTBI. At the same time, among those soldiers who reported in-theatre that they had no history of mTBI and who later reported mTBI post-deployment (No TBI-TBI), it is certainly conceivable that the $T_{2}$ endorsements were the more reliable report. As noted previously, some veterans who undergo screening before leaving the combat zone may minimise combat-related injuries, such as mTBI, in the interest of maintaining unit cohesion, to fulfil the perceived expectations of their peers or superior officers or to avoid delays in completing the postdeployment assessment process. ${ }^{11}$ Even though participants were informed that their survey responses would remain confidential and not be shared with command, some soldiers may have chosen not to acknowledge mTBI histories until they had successfully completed their tours of military service and returned home. However, this seems unlikely, since many of these same soldiers acknowledged psychological symptoms in-theatre.

It is also possible that soldiers with greater post-deployment trauma and other psychiatric symptoms may have been more likely to seek services through the Veterans Affairs system of care. Coincident with obtaining Veterans Affairs care, veterans may have received education relevant to $\mathrm{mTBI}$, which in turn allowed them to more accurately identify a previous combat event that contributed to mTBI. Finally, it is possible that at least a portion of the current sample that endorsed mTBI at post-deployment, but not at the time they were surveyed in-theatre, sustained injuries within the year after their return from Iraq and failed to appreciate that injuries sustained during their recent deployment were to be reported. In fact, one recent longitudinal investigation of neuropsychological outcomes in OEF/OIF personnel ${ }^{30}$ identified that although most head injuries transpired during deployment, as many as $21 \%$ of reported injuries were sustained outside the context of deployment.

Various cultural dynamics and social influences that veterans encounter during the post-deployment phase might also reinforce or heighten expectations that persisting symptoms reflect a history of mTBI. Therefore, changes in respondents' self-reports of mTBI over time (reporting no mTBI at $T_{1}$ and endorsing mTBI at $T_{2}$ ) might reflect misattribution of psychological and emotional difficulties to mTBI related to concerns of social stigmatisation surrounding mental health diagnoses, and a related preference for physical as opposed to psychological explanations for their difficulties. ${ }^{31}$ During the post-deployment transition, some veterans might receive false information regarding the nature of mTBI and expectations for symptom recovery, and falsely ascribe mental health issues to an historical mTBI. Veterans who are not aware of the natural history of mTBI, which typically involves a rapid and favourable course of recovery in the majority of samples, ${ }^{32}$ might be especially vulnerable to social influence and development of the false expectation that their symptoms are a result of mTBI in the year after their return from deployment.

Procedures within the Veterans Affairs system of care itself might also inadvertently contribute to false expectations regarding mTBI history and account for changes in post-deployment endorsement. For example, as well intended as the administration of TBI screening instruments as currently practiced by Department of Defense and Veterans Health Administration may be, various researchers ${ }^{33,34}$ have suggested that this practice is potentially iatrogenic and inadvertently contributes to the false expectation that symptoms relate to remote mTBI. One especially compelling commentary ${ }^{34}$ has recently suggested that routine TBI screening as currently practised is not only unnecessary, but potentially harmful.

\section{Limitations}

Several limitations should be considered. First, as with most survey research that has been conducted in this area, ${ }^{1,2}$ we did not have regular access to acute-stage injury medical records or other collateral information that might inform the plausibility that mTBI was in fact sustained at any time during combat. Therefore, any conceptualisation of the current findings should be made only with the understanding that one cannot definitively determine which endorsement of mTBI, that obtained at $T_{1}$ or $T_{2}$, is the more reliable representation of actual events confronted during combat. Another important limitation of the current study relates to how mTBI was operationalised. By convention, ${ }^{4} \mathrm{mTBI}$ is rated not only on the basis of loss of consciousness/altered mental status, but also post-traumatic amnesia duration and acute-stage neurological symptoms. Further, although relatively few studies have in fact examined the test-retest reliability of self-reported mTBI according to loss of consciousness, it appears that reliability of self-reported mTBI is attenuated as the interval between injury and report increases. Illustrating this point, whereas test-retest reliability of self-reported mTBI was relatively strong among veterans who completed the Veterans Affairs clinical reminder within a 2 -week interval, ${ }^{14}$ test-retest reliability was quite poor when a 6-month interval transpired between assessments. ${ }^{15}$ The interval between $T_{1}$ and $T_{2}$ in the current study was even longer (approximately 1 year). As such, it is conceivable that some veterans interpreted altered mental status and related physiological changes following a given combat event (for example, blast exposure) 
to psychological trauma (such as autonomic dysregulation associated with the stress response) at $T_{1}$, and interpreted the same altered mental status and physiological changes to physical trauma (such as mTBI) at $T_{2}$. Items used to assess loss of consciousness/altered mental status also varied subtly between times of screening; it is possible that inconsistencies partially reflect measurement error, although this issue is unlikely to fully account for inconsistent reports of mTBI in the current sample as a significantly greater number of veterans responded in one direction (i.e. denial of mTBI at $T_{1}$ with endorsement of mTBI at $T_{2}$ and not vice versa). Further, similar to previous studies, ${ }^{1,2}$ physical and psychological outcomes in the current study were restricted to relatively brief and face valid questionnaires and symptom checklists. Self-report questionnaires tend to be vulnerable to self-report bias, ${ }^{35,36}$ and individuals with histories of mTBI tend to endorse a greater number of symptoms on questionnaires relative to clinical interviews that consist of open-ended questioning. ${ }^{35}$ Finally, the PHQ was administered at $T_{2}$ only, which restricts the ability to understand the extent to which non-specific physical health complaints experienced in-theatre may have been associated with inconsistencies in self-reported mTBI over time.

\section{Implications}

Current findings have implications for physicians, psychologists, rehabilitation specialists and other clinicians who provide care on behalf of military service members and veterans returning from OEF/OIF/OND with self-reported histories of mTBI. Clinicians should be encouraged by the current findings that show the vast majority of veterans remained consistent in their endorsement of mTBI across time. In particular, it should be appreciated that most veterans who deny a history of mTBI at $T_{2}$ are consistent in their self-reported mTBI status over time. This might suggest that the accounts of those who deny a history of combat-related mTBI at $T_{2}$ may be more reliable over time and, as such, their self-reported histories of mTBI may not require the same level of scrutiny as those who do endorse a history of mTBI at $T_{2}$.

Still, findings do point to concerns that a meaningful minority of veterans show discrepancies in their responses to screening questions about mTBI in the year after returning from deployment, the same time frame in which Veterans Affairs providers are most likely to first evaluate and treat veterans. Remarkably, in the current study nearly two-thirds ( $n=123 / 195$, $63.1 \%$ ) of veterans who endorsed mTBI histories in the year after return from deployment $\left(T_{2}\right)$ denied mTBI history in the month before returning to the USA $\left(T_{1}\right)$, a time that was more proximate to combat activity. These results suggest that clinicians cannot assume that Veterans' post-deployment endorsements of mTBI are necessarily reliable or accurate portrayals of their previous combat experiences. Moreover, the presence of emotional and physical distress in the year after returning from the combat zone increases the likelihood of changes in self-reports of mTBI.

Therefore, clinicians are encouraged to use great discretion when interpreting veterans' subjective descriptions of previous combat injuries and conceptualising the most likely source(s) of persisting symptoms. As noted, Veterans Affairs providers rarely have access to medical records and other collateral sources of information that might corroborate reported combat histories. ${ }^{9}$ Nevertheless, to the degree possible, clinicians are encouraged to augment self-report information with additional sources (for example medical technician reports; eyewitness accounts) to inform the likelihood that concussive injury was plausibly sustained. Such investigation may ensure that veterans receive treatment interventions that are appropriate to their ongoing difficulties (for example psychotherapy and other mental health treatments) and simultaneously prevent the unnecessary 'rehabilitation' of a TBI that may or may not have been sustained during combat. Irrespective of reporting reliability or accuracy, research consistently demonstrates that a remote history of combat-related mTBI as such is unlikely to account for the full extent of symptoms or impairments that persist in the months and years that follow deployment. ${ }^{32}$ Clinicians are advised to provide routine early education ${ }^{37}$ and encouragement regarding the favourable recovery that is anticipated following mTBI in the interest of dismantling cognitive biases and misattribution processes that may underlie veterans' delayed endorsement of mTBI.

It should also be recognised that in spite of the statistical differences that were demonstrated on measures of PTSS and physical symptoms at $T_{2}$, the majority of participants did not endorse symptoms to a degree that would necessarily reach a threshold of clinical significance, regardless of mTBI status. $T_{2}$ PCL symptoms, for example, although statistically significant between the No TBI-No TBI and No TBI-TBI groups, were nevertheless below common cut-scores that are often regarded to be of diagnostic significance.

Current results also have implications for researchers who conduct studies with OEF/OIF mTBI samples. Typically, researchers, like clinicians, do not have complete access to acute-stage of injury information that confirms the reliability of self-reported mTBI. In spite of the unknown reliability of selfreport information, researchers typically rely heavily on retrospective accounts of previous combat events to define mTBI membership. The limited consistency of self-reported mTBI among a significant proportion of the current sample brings into question the interpretability of research findings on mTBI in OEF/OIF/OND samples. Some research suggests, for example, that combat-related mTBI increases risk of PTSD and other psychological difficulties in OEF/OIF service members. ${ }^{1}$ The lack of consistency in selfreported mTBI among a subsample of respondents in the current study would suggest that researchers may not be able to determine whether self-reported mTBI increases risk of post-deployment distress, or post-deployment distress increases risk of self-reported mTBI. This question warrants further research investigation.

The categorical assignment of participants to 'mTBI' groups on the basis of near exclusive self-report information also raises questions about the interpretability of other recent studies conducted in OEF/OIF/OND samples, such as those that examine neuropsychological outcomes ${ }^{38}$ among veterans with self-reported histories of combat-related mTBI. Current results suggest that a substantial number of 'mTBI' participants included in this study would not have endorsed a history of mTBI had they been surveyed at an earlier point in time. The interpretability of any study that contrasts outcomes according to mTBI status is likely attenuated by the limited reliability of self-reported mTBI in the post-deployment phase.

Our findings should also be considered in conjunction with other recent surveys of mTBI, PTSD and outcomes of deployment samples from other world regions. Our findings are especially relevant to a recent survey of 1363 British military personnel deployed to Afghanistan in 2011. ${ }^{39}$ Like the current study, the authors surveyed participants 1 month prior to their completion of deployment regarding injuries and symptoms associated with different combat events (for example blast exposure). Although relatively fewer British personnel endorsed at least one potential mTBI during deployment (5.9\%, compared with $9 \%$ at $T_{1}$ in the current sample), the authors found a significant association between PTSD symptoms and endorsement of combat-related mTBI (and 'mTBI symptoms'). Taking their findings ${ }^{39}$ and the current findings together, it would seem that regardless of the country of origin (USA or UK) or the region of deployment 
(Iraq or Afghanistan), PTSD symptoms significantly influence perceptions of combat-related mTBI and potentially confound the ability to discriminate the source (for example physical $v$. psychological trauma event) of symptoms that follow from combat activity.

Regardless of the true source of the inconsistent endorsement of mTBI that were observed in the current study, whether it relates to minimisation of mTBI histories in-theatre, post-deployment misattribution of non-specific symptoms related to distress, social and cultural influence factors or any admixture of these factors during the post-deployment period, results show that a significant minority of veterans who endorsed a history of combat-related mTBI during the post-deployment phase did not endorse a history of $\mathrm{mTBI}$ at a time more proximate to injury. Clinicians, researchers and policy-makers alike should be mindful that persisting physical, psychological and emotional symptoms cannot necessarily be reliably linked with self-reported history of mTBI itself; results suggest that post-deployment distress and physical complaints are associated with inconsistent reports of combat-related mTBI. Until future researchers effectively integrate external documentation of acute-stage injury characteristics with late-stage accounts of mTBI, ideally through a Department of Defense/Veterans Health Administration collaboration, the true prevalence of $\mathrm{mTBI}$ and the potential independent contribution of $\mathrm{mTBI}$ on intermediate and primary functional outcomes among OEF/OIF veterans will remain uncertain.

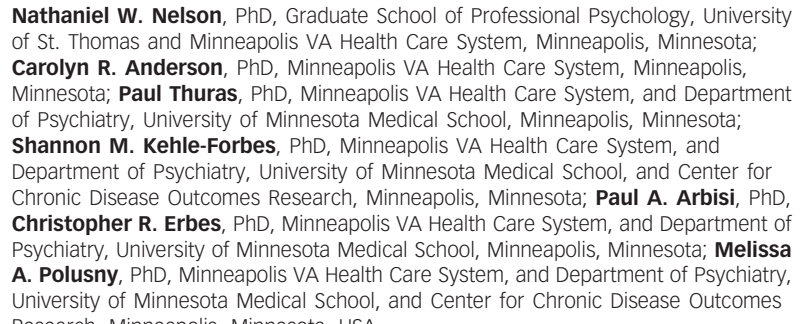

First received 29 Mar 2014, final revision 31 Jul 2014, accepted 21 Aug 2014

\section{Funding}

This material is based on work supported by the Department of Veterans Affairs, Veteran Health Administration, Office of Research and Development (Quality Enhancement Research Initiative Rapid Response Project 08-252) and the resources and facilities of the Minneapolis VA Health Care System. The views expressed in this manuscript are those of the authors and do not reflect the official policy or position of the Department of Veterans Affairs, the Department of Defense or the US Government. S.M.K.-F. is supported by a VA Health Services Research \& Development Career Development Award.

\section{Acknowledgements}

The authors gratefully acknowledge the men and women in the National Guard who participated in this study as well as Colonel (Retired) Michael Rath, MD and Major Cora Courage, PsyD, for their assistance with this project.

\section{References}

1 Hoge CW, McGurk D, Thomas JL, Cox AL, Engel CC, Castro CA. Mild traumatic brain injury in U.S. soldiers returning from Iraq. N Engl J Med 2008; 358: 453-63.

2 Schneiderman Al, Braver ER, Kang HK. Understanding sequelae of injury mechanisms and mild traumatic brain injury incurred during the conflicts in Iraq and Afghanistan: persistent postconcussive symptoms and posttraumatic stress disorder. Am J Epidemiol 2008; 167: 1446-52.
3 Polusny MA, Kehle, SM, Nelson NW, Erbes CR, Arbisi PA, Thuras P. Longitudinal effects of mild TBI and PTSD comorbidity on post-deployment outcomes in National Guard soldiers deployed to Iraq. Arch Gen Psychiatry 2011; 68: 79-89.

4 Kay $\mathrm{T}$, Harrington $\mathrm{DE}$, Adams, R, Anderson, T, Berrol, S, Cicerone $\mathrm{K}$, et al. Definition of mild traumatic brain injury. J Head Trauma Rehab 1993; 8: 86-87.

5 DePalma RG, Burris DG, Champion HR, Hodgson MJ. Blast injuries. N Engl J Med 2005; 352: 1335-42.

6 Tanielian T, Jaycox LH. Invisible Wounds of War: Psychological and Cognitive Injuries, their Consequences, and Services to Assist Recovery. RAND Corporation, 2008

7 Terrio H, Brenner LA, Ivins BJ, Cho JM, Schwab K, Scally K, et al. Traumatic brain injury screening: preliminary findings in a U.S. Army Brigade combat team. J Head Trauma Rehabil 2009; 24: 14-23.

8 Terrio HP, Nelson LA, Betthauser LM, Harwood JE, Brenner LA. Postdeployment traumatic brain injury screening questions: sensitivity, specificity, and predictive values in returning soldiers. Rehabil Psychol 2011; 56: 26-31.

9 Belanger HG, Uomoto JM, Vanderploeg RD. The Veterans Health Administration system of care for mild traumatic brain injury: costs, benefits, and controversies. J Head Trauma Rehabil 2009; 24: 4-13.

10 General Accounting Office. VA Health Care. Mild Traumatic Brain Injury Screening and Evaluation Implemented for OEF/OIF veterans, but Challenges Remain. GAO-08-276. General Accounting Office, 2008 (http://www.gao.gov/ assets/280/271988.pdf).

11 Iverson GL, Langlois JA, McCrea MA, Kelly JP. Challenges associated with post-deployment screening for mild traumatic brain injury in military personnel. Clin Neuropsychol 2009; 23: 1299-314.

12 Schwab KA, Ivins B, Cramer G, Johnson W, Sluss-Tiller M, Kiley K, et al. Screening for traumatic brain injury in troops returning from deployment in Afghanistan and Iraq: initial investigation of the usefulness of a short screening tool for traumatic brain injury. J Head Trauma Rehabil 2007; 22: 377-89.

13 Belanger HG, Vanderploeg RD, Soble JR, Richardson M, Groer S. Validity of the Veterans Health Administratin's traumatic brain injury screen. Arch Phys Med Rehabil 2012; 93: 1234-39.

14 Donnelly KT, Donnelly JP, Dunnam M, Warner GC, Kittelson CJ, Constance JE, et al. Reliability, sensitivity, and specificity of the VA traumatic brain injury screening tool. J Head Trauma Rehabil 2011; 26: 439-53.

15 Van Dyke SA, Axelrod BN, Schutte C. Test-retest reliability of the traumatic brain injury screening instrument. Mil Med 2010; 175: 947-49.

16 Panayiotou A, Jackson M, Crowe SF. A meta-analytic review of the emotional symptoms associated with mild traumatic brain injury. JCEN 2010; 32 : 463-473.

17 Wessely S, Unwin C, Hotopf M, Hull L, Ismail K, Nicolaou V, et al. Stability of recall of military hazards over time: evidence from the Persian Gulf War of 1991. Br J Psychiatry 2003; 183: 314-22.

18 Heir T, Piatigorsky A, Weisaeth L. Longitudinal changes in recalled perceived life threat after a natural disaster. Br J Psychiatry 2009; 194: 510-4.

19 Engelhard IM, van den Hout MA, McNally RJ. Memory consistency for traumatic events in Dutch soldiers deployed to Iraq. Memory 2008; 16 3-9.

20 David A-C, Akerib V, Gaston L, Brunet A. Consistency of retrospective reports of peritraumatic responses and their relation to PTSD diagnostic status. J Traumatic Stress 2010; 23: 599-605.

21 Clauw DJ, Engel CC, Aronowitz R, Jones E, Kipen HM, Kroenke K, et al. Unexplained symptoms after terrorism and war: An expert consensus statement. J Occup Environ Med 2003; 45: 1040-48.

22 Howe LLS. Giving context to post-deployment post-concussive-like symptoms: blast-related potential mild traumatic brain injury and comorbidities. Clin Neuropsychol 2009; 23: 1315-37.

23 Nelson NW, Hoelzle JB, McGuire KA, Ferrier-Auerbach AG, Charlesworth MJ, Sponheim SR. Evaluation context impacts neuropsychological performance of OEF/OIF veterans with reported combat-related concussion. Arch Clin Neuropsychol 2010; 25: 713-23.

24 Nelson NW, Hoelzle JB, McGuire KA, Sim AH, Goldman DJ, Ferrier-Auerbach AG, et al. Self-report of psychological function among OEF/OIF personnel who also report combat-related concussion. Clin Neuropsychol 2011; 25: 716-40.

25 Bush SS, Graver CJ. Symptom validity assessment of military and veteran populations following mild traumatic brain injury. In Mild Traumatic Brain Injury; Symptom Validity Assessment and Malingering (eds DA Carone and SS Bush): 381-97. Springer, 2013.

26 Weathers FW, Litz BT, Herman DS, Huska JA, Keane TM. The PTSD Checklist (PCL-M): Reliability, Validity, and Diagnostic Utility. Paper presented at Annual 
Meeting of the International Society for Traumatic Stress Studies, 1993 (available at http://www.pdhealth.mil/guidelines/appendix4.asp).

27 Beck AT, Steer RA, Brown GK. Manual for the Beck Depression Inventory (2nd edn). Psychological Corporation, 1996

28 Spitzer RL, Kroenke K, Williams JB. Patient Health Questionnaire Primary Care Study Group Validation and utility of a self-report version of PRIME-MD: the PHQ-15 primary care study. JAMA 1999; 282: 1737-44.

29 WHOQOL Group. Development of the World Health Organization WHOQOLBREF quality of life assessment. Psychol Med 1998b; 28: 551-8.

30 Vasterling JJ, Brailey $\mathrm{K}$, Proctor SP, Kane R, Heeren T, Franz M. Neuropsychological outcomes of mild traumatic brain injury, post-traumatic stress disorder and depression in Iraq-deployed US Army soldiers. Br J Psychiatry 2012; 201: 186-92.

31 Brenner LA, Vanderploeg RD, Terrio H. Assessment and diagnosis of mild traumatic brain injury, posttraumatic stress disorder, and other polytrauma conditions: burden of adversity hypothesis. Rehabil Psychol 2009; 54: 239-246.

32 Iverson GL. Outcome from mild traumatic brain injury. Curr Opin Psychiatry 2005; 18: 301-17.

33 Fear NT, Jones E, Groom M, Greenberg N, Hull L, et al. Symptoms of postconcussional syndrome are non-specifically related to mild traumatic brain injury in UK armed forces personnel on return from deployment in Iraq: an analysis of self-reported data. Psychol Med 2009; 39: 1379-87.

34 Vanderploeg RD, Belanger HG. Screening for a remote history of mild traumatic brain injury: when a good idea is bad. J Head Trauma Rehabil 2013; 28: $211-8$.

35 Iverson GL, Brooks BL, Ashton VL, Lange RT. Interview versus questionnaire symptom reporting in people with the postconcussion syndrome. I Head Trauma Rehabil 2010; 25: 23-30.

36 Maestas KL, Benge JF, Pastorek NJ, LeMaire A, Darrow R. Factor structure of posttraumatic stress disorder symptoms in OEF/OIF veterans presenting to a polytrauma clinic. Rehabil Psychol 2011; 56: 366-73.

37 Veterans Affairs/Department of Defense. VA/DoD Clinical Practice Guideline Management of Concussion/Mild Traumatic Brain Injury. Veterans Affairs/ Department of Defense, 2009.

38 Nelson NW, Hoelzle JB, Doane BM, McGuire KA, Ferrier-Auerbach AG, Charlesworth MJ, et al. Neuropsychological outcomes of U.S. Veterans with report of remote blast-related concussion and current psychopathology. J Int Neuropsychol Soc 2012; 18: 845-55.

39 Jones N, Fear NT, Rona R, Fertout M, Thandi G, Wessely S, et al. Mild traumatic brain injury (mTBI) among UK military personnel whilst deployed in Afghanistan in 2011. Brain Injury 2014; 28: 896-99.

\section{Locked in}

\section{Andrew Gardner}

Few medical students get the opportunity to experience a psychiatric intensive care unit (PICU) during their training. I had little idea what to expect - my best guess was some very physically ill patients who also happened to be psychotic. For some reason, it never crossed my mind it would be the nature of the patients' psyche that would denote their place of institution. Perhaps this is because as medical students, or even the social group from which we usually arise, we have little exposure to these extremes of mental illness.

My naivety made my first impression of the ward all the more surreal: I could not quite grasp the point of it all. Most of the time nurses just stood around with their patients, until every so often one of the patients 'kicked off' and the nurses restrained each available limb, administered sedating medication and escorted them into another, slightly more Spartan (de-escalation) room, until they cooled down. Stern words were juxtaposed with reassurance. The doctors were hands off, directing changes in medication and mostly suggesting patients' management from afar. What were we actually doing here, if not just acting as childminders? Were these patients even really ill? It took me until my fourth and final week to understand just how wrong my interpretation was. At first glance, compassion is easy to miss in PICU.

I have learnt that a great majority of psychiatry is about re-forging relationships; be it those between the ideas darting around a patient's mind or with the individuals around them. While doctors contribute diagnosis, prognosis and a leadership in such an unpredictable spectrum of disease, it is primarily nurses who manage patients' illness day to day. They constantly challenge and redirect misaligned thoughts, the effect of which pharmacology can usually only serve to dampen or numb. As medical students our only true requirement is to observe, interview and present patients for exam practice and our own psychiatric curiosity: a shadow of our future role. Yet, a nursing student is expected to act as if they were a nurse, with full-time responsibilities. This difference undermines an education in the interdisciplinary nature of psychiatry. As a student, without immersion in the constant dialogue of patient care, the subtlety of psychiatry is lost.

Psychiatry is a specialty which must embrace multidisciplinary care and champion it for the National Health Service. Interdisciplinary teamwork should also be reflected in student training, a move which will serve to degrade the old barriers of tribalism, which are often still evident in healthcare today. For example, each medical student could be attached to a nurse mentor and should be encouraged, if not compelled, to contribute towards patient care. Without a proper understanding of how to manage psychiatric illness, if not now, then how as an F1 in A\&E? I went into PICU wanting psychiatry to have simple solutions, which in my mind involved various coloured pills targeting well-defined disease. Now I realise, perhaps that's not as easy as it seems. 